\title{
A Bayesian Multi-Scale Framework for Photoplethysmogram Imaging Waveform Processing
}

Brendan Chwyl

Robert Amelard

David A. Clausi

Alexander Wong
University of Waterloo, ON, Canada

University of Waterloo, ON, Canada

University of Waterloo, ON, Canada

University of Waterloo, ON, Canada

\section{Abstract}

Photoplethysmography imaging (PPGI) is an increasingly popular technique for remotely creating signals with a plethora of medical information, referred to as PPGI waveforms. However, PPGI waveforms are often heavily affected by illumination variation and motion artefacts. Current PPGI waveform processing methods are useful for estimating heart rate, however, structural detail is not preserved, rendering the signal incapable of providing additional medical information. For this reason, we propose a multi-scale framework based on the Bayesian residual transform which aims to suppress noise and preserve structural details necessary for extracting cardiovascular information beyond the scope of heart rate. Experiments conducted on a dataset consisting of 24 different PPGI waveforms and corresponding PPG waveforms captured via a finger pulse oximeter suggests a high level of noise and ambient illumination variation suppression is achieved while signal fidelity is largely retained.

\section{Introduction}

The measurement of blood volume as it varies over time is critical for non-invasive heart rate measurement devices such as pulse oximeters. This measurement is referred to as a photoplethysmograph (PPG) waveform and is acquired by measuring the absorption of light through a thin extremity (e.g., finger tip, ear lobe). Due to the optical absorption spectrum of hemoglobin, a protein present in blood, the amount of light absorbed in certain wavelengths varies depending on blood volume, allowing blood volume to be inferred from optical measurements.

While PPG waveforms are invaluable to heart rate estimation, the analysis of detailed PPG waveforms can also provide insight into cardiac output, vasomotor tone, and fluid loading [1]. Though PPG waveforms acquired from pulse oximeters are typically clean enough for analysis, there is a growing trend in constructing PPG waveforms remotely via camera systems due to improvements in cleanliness, comfort, and convenience. This technique is referred to as photoplethysmogram imaging (PPGI) and the waveforms captured via PPGI, referred to here as PPGI waveforms, are contaminated with a high degree of noise due to motion artefacts, ambient illumination changes, and relatively low signal strength. A comparison of a PPG waveform acquired from a finger pulse oximeter and a PPGI waveform acquired from a near-infrared video recording can be seen in Fig 1, where the signal fidelity of the PPGI waveform is significantly lower than the PPG waveform. Subsequently, detrending and smoothing techniques are often applied to clean the PPGI waveform such that heart rate can be estimated; however, signal fidelity is often lost in the process, making it difficult to extract detailed cardiovascular information.

Typical PPGI systems aim to estimate heart rate and are primarily concerned with the frequency response of the acquired PPGI waveforms. The PPGI waveforms are often transformed into the frequency domain via the Fourier transform prior to being bandpass filtered to reject frequencies out of the scope of reasonable heart rates. Though this has proven to be an effective technique for heart rate estimation, high frequency components corresponding to structural detail in the time domain are lost, and using the processed PPGI waveform for measurements beyond heart rate becomes infeasible. While PPG waveform processing techniques aimed at preserving structural detail exist, motion artefacts and ambient illumination changes introduced by PPGI systems make these methods unsuitable for processing PPGI waveforms.

We propose a multi-scale signal processing framework based on the Bayesian residual transform (BRT) as a means of processing a PPGI waveform while retaining signal fidelity. In addition, this framework does not require a band of reasonable heart rates and is subsequently more robust to heart rates which fall above or below the cut-off frequencies of bandpass filter methods.

\section{Methods}

In this section, a multi-scale signal processing framework based on the Bayesian residual transform (BRT) is described. The proposed framework consists of three components. First, detrending is performed by removing the low frequency components of the raw PPGI waveform. Second, the BRT [5] is applied to decompose the PPGI waveform into multiple residual signals at different scales. Lastly, the noise within each residual signal is suppressed and a noise suppressed PPGI is constructed via the inverse BRT.

\subsection{Detrending}

To compensate for ambient illumination changes, the low frequency components of the raw PPGI signal are suppressed. This is accomplished via a smoothness of priors detrending method described by Tarvainen et al. [3]. This method was selected as it is specifically designed for processing cardiac signals (e.g., PPG waveforms, PPGI waveforms, and ECG signals). The effects of detrending a PPGI waveform can be seen in Fig. 2 .

\subsection{Bayesian Residual Transform}

The Bayesian Residual Transform [5] (BRT) aims to decompose a signal into multiple residual signals, where each residual signal represents information at a different scale. This can be represented as

$$
\begin{gathered}
B(\phi(t))=\left\{r_{1}(t), r_{2}(t), \ldots, r_{n}(t)\right\} \\
B^{-1}\left(r_{1}(t), r_{2}(t), \ldots, r_{n}(t)\right)=\phi(t)=\sum_{i=1}^{n} r_{i}(t)
\end{gathered}
$$

where $B(\phi(t))$ is the BRT performed on a PPGI waveform, $\phi(t)$, $r_{i}(t)$ is a residual signal containing information at the $i^{t h}$ scale, and $B^{-1}$ is the inverse BRT. Let $\phi_{\Sigma, j}(t)$ represent the summation of all residual signals from signal $j$ to signal $n$ :

$$
\phi_{\Sigma, j}(t)=\sum_{i=j}^{n} r_{i}
$$

The $i^{\text {th }}$ residual signal can then be calculated as

$$
r_{i}(t)=\phi_{\Sigma, i}(t)-\phi_{\Sigma, i+1}(t) .
$$

Each increasing scale can be treated as an inverse problem of estimating $\phi_{\Sigma, i+1}(t)$ given $\phi_{\Sigma, i}(t)$ :

$$
\hat{\phi}_{\Sigma, i+1}(t)=E\left(\phi_{\Sigma, i+1}(t) \mid \phi_{\Sigma, i}(t)\right),
$$

where $\hat{\phi}_{\Sigma, i+1}(t)$ is an estimate of $\phi_{\Sigma, i+1}(t)$ and $E($.$) is the conditional$ expectation. Practically, residual signals can be calculated by iteratively solving Eq. 5 via kernel regression, a technique described in detail by Wong et al. [5].

\subsection{Noise Suppression}

To generate a noise suppressed PPGI waveform, $\hat{\phi}(t)$, from the estimated residual signals, noise is suppressed from each individual residual signal prior to performing the inverse BRT. The noise suppression for the $i^{t h}$ residual signal, $r_{i}(t)$, is formulated as

$$
\hat{r}_{i}(t)= \begin{cases}0 & \left|r_{i}(t)\right|<\theta_{i} \\ r_{i}(t) & \text { otherwise }\end{cases}
$$

where $\theta_{i}$ is a threshold calculated based on the seminal noise. The seminal noise is computed as 
Table 1: Tabulated averages $\left(M_{\text {corr }}\right)$ and standard deviations $\left(\sigma_{\text {corr }}\right)$ of Pearson correlation values calculated for PPGI waveforms processed with the proposed framework, as well as the raw PPGI waveform and detrended PPGI waveform.

\begin{tabular}{|l|c|}
\hline & $M_{\text {corr }}\left(\sigma_{\text {corr }}\right)$ \\
\hline Proposed & $0.62(0.13)$ \\
Detrended & $0.38(0.10)$ \\
Raw & $0.22(0.10)$ \\
\hline
\end{tabular}

$$
\theta_{i}=\frac{M A D\left(r_{i}(t)\right)}{\Phi^{-1}(3 / 4)}
$$

where $M A D($.$) is the median absolute deviation and \Phi^{-1}$ is the normal inverse cumulative distribution function. The noise suppressed PPGI waveform can then be generated as

$$
\hat{\phi}(t)=B^{-1}\left(\hat{r}_{1}(t), \hat{r}_{2}(t), \ldots, \hat{r}_{n}(t)\right) .
$$

\section{Experiments}

\subsection{Experimental Setup}

To verify the effectiveness of our method, we use a near-infrared (NIR) dataset collected by Amelard et al. [4]. This dataset consists of near-infrared video and finger pulse oximeter PPG waveform recordings for 24 different participants laying still in a supine position. From each video, a PPGI waveform was constructed via the method described by Chwyl et al. [2]. Because the ground truth PPG waveform is obtained via a finger pulse oximeter, the constructed PPG waveforms are a measure of blood volume absorption. Since the PPGI waveforms are constructed from near infrared video recordings, the resulting signals are based on hemoglobin reflectance. Therefore, it is necessary to convert the PPGI waveforms from reflectance to absorption to provide a proper comparison to the ground truth PPG waveforms. The PPGI waveform is converted into absorption via the Beer-Lambert law:

$$
\phi_{a}(t)=-\ln \left(\phi_{r}(t)+1\right),
$$

where $\phi_{a}(t)$ is the PPGI waveform in terms of absorption and $\phi_{r}(t)$ is the normalized PPGI waveform in terms of reflectance. The resulting absorption PPGI waveforms were then processed by the framework described in this paper. Residual signals at eight different scales were used in the BRT processing step as this value was empirically determined to produce favourable results.

\subsection{Experimental Results}

To offer quantitative results, the Pearson correlation between each processed PPGI waveform and corresponding PPG waveform was

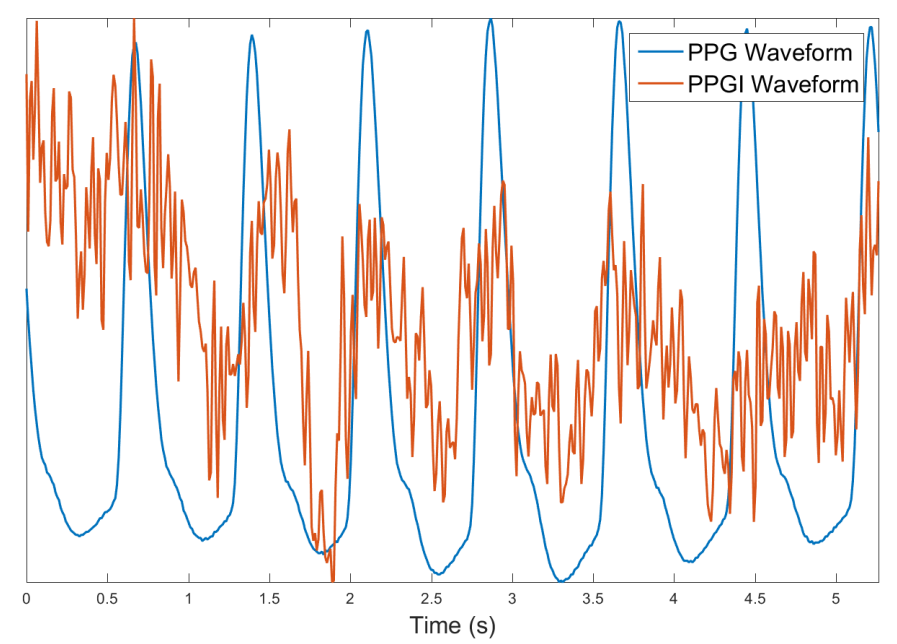

Fig. 1: An example of a PPG waveform acquired from a finger pulse oximeter versus a PPGI waveform acquired via a near infrared video video recording.

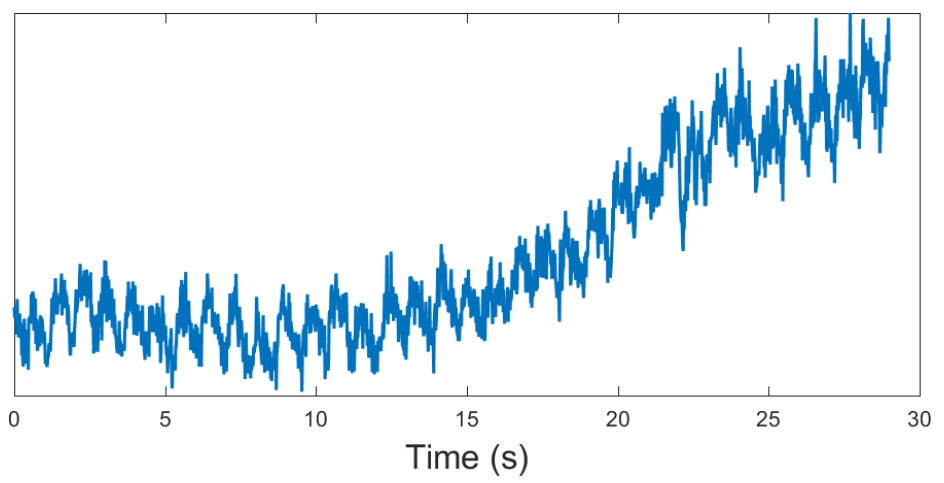

(a) Raw PPGI waveform

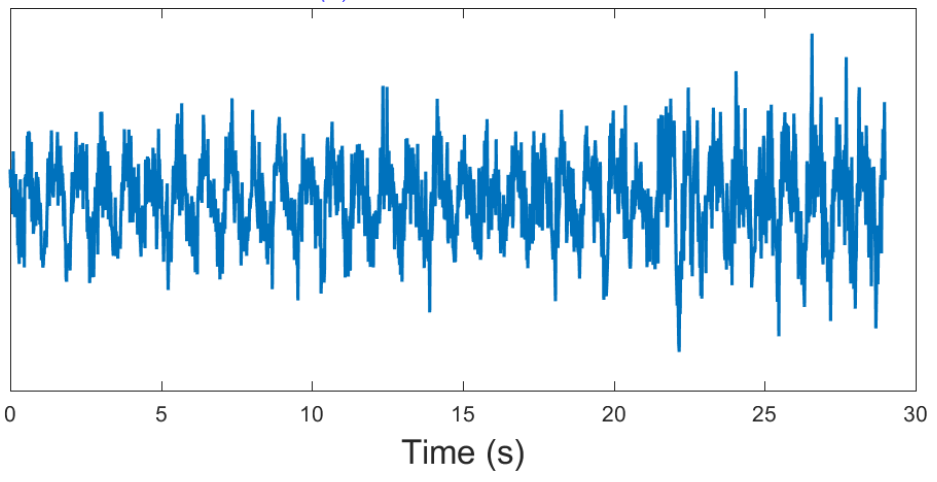

(b) Detrended PPGI waveform

Fig. 2: Comparison of a raw PPGI waveform (A) and a detrended PPGI waveform (B).

calculated. The same metric was also calculated for the raw PPGI waveforms and for the detrended PPGI waveforms, once again using the ground truth PPG waveforms as the reference. The average and standard deviation of the resulting Pearson correlations are tabulated in Table 1 where a value of 1.0 indicates perfect correlation, negative 1.0 indicates perfect negative correlation, and a value of 0 indicates no correlation. It can be seen that the proposed framework improves correlation, however, the correlation remains relatively low. Although the PPG waveforms offer a reference for quantitative analysis, discrepancies between the PPGI waveforms and PPG waveforms are expected as these signals were captured at different locations on the body (cheek region versus finger tip) as well as via different modalities (near-infrared video versus finger pulse oximeter). For this reason, qualitative analysis was also performed.

A subsection of results can be seen in Fig 3. We observe that the proposed method typically retains structural detail of the waveform and suppresses most noise. In particular, individual pulses often retain both the primary peak, referred to as the systolic peak, as well as the subsequent peak, referred to as the dicrotic notch. The location of the dicrotic notch is useful in the estimation of vasomotor tone; a metric which can give indication to increased blood pressure.

\section{Conclusions}

A multi-scale framework designed to process PPGI waveforms was proposed, with the aim to suppress noise and ambient illumination variations while pre- serving medically relevant structures. Quantitative results indicate an improved level of correlation between the ground truth PPG waveform and the processed PPGI waveform. Qualitative analysis shows a high level of noise suppression while structural detail was retained. Future works will include a more comprehensive comparison between the proposed method and current state-of-the-art PPG processing methods as well as exploring the feasibility of using the resulting PPGI waveforms to estimate vasomotor tone.

\section{Acknowledgments}

This research was undertaken, in part, thanks to funding from the Canada Research Chairs program. The study was also funded by 


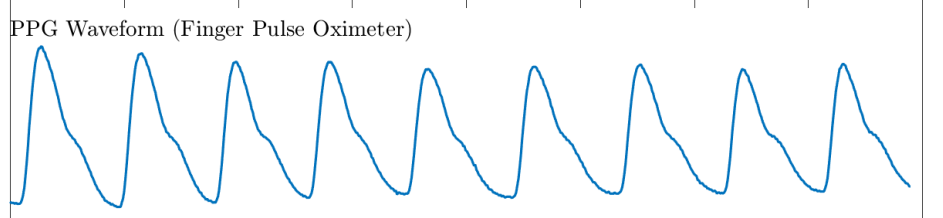

Detrended PPGI Waveform
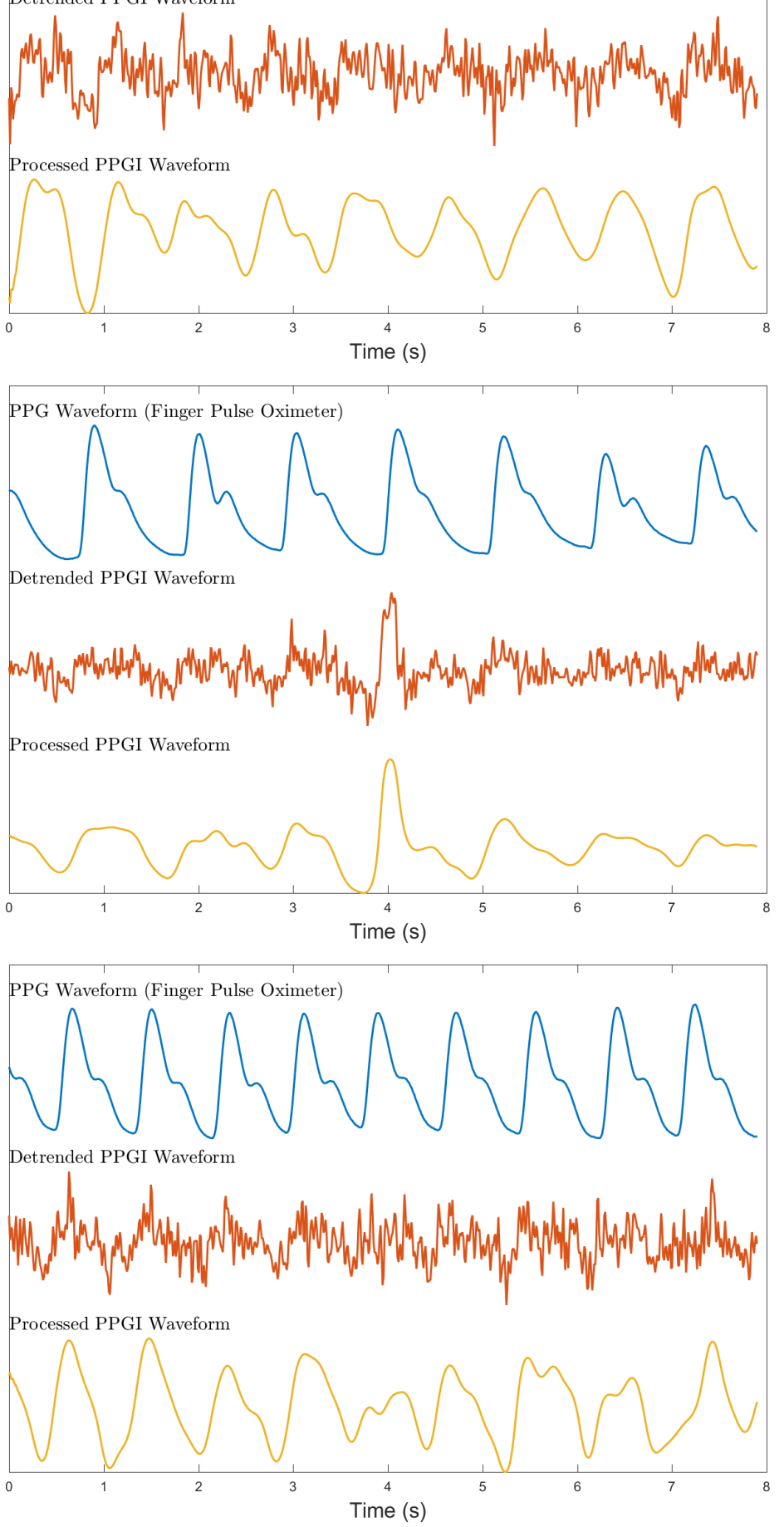

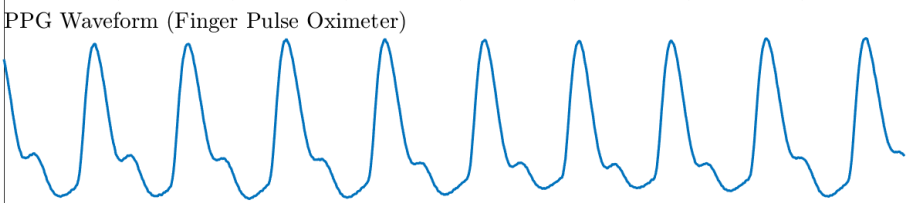

Detrended PPGI Waveform
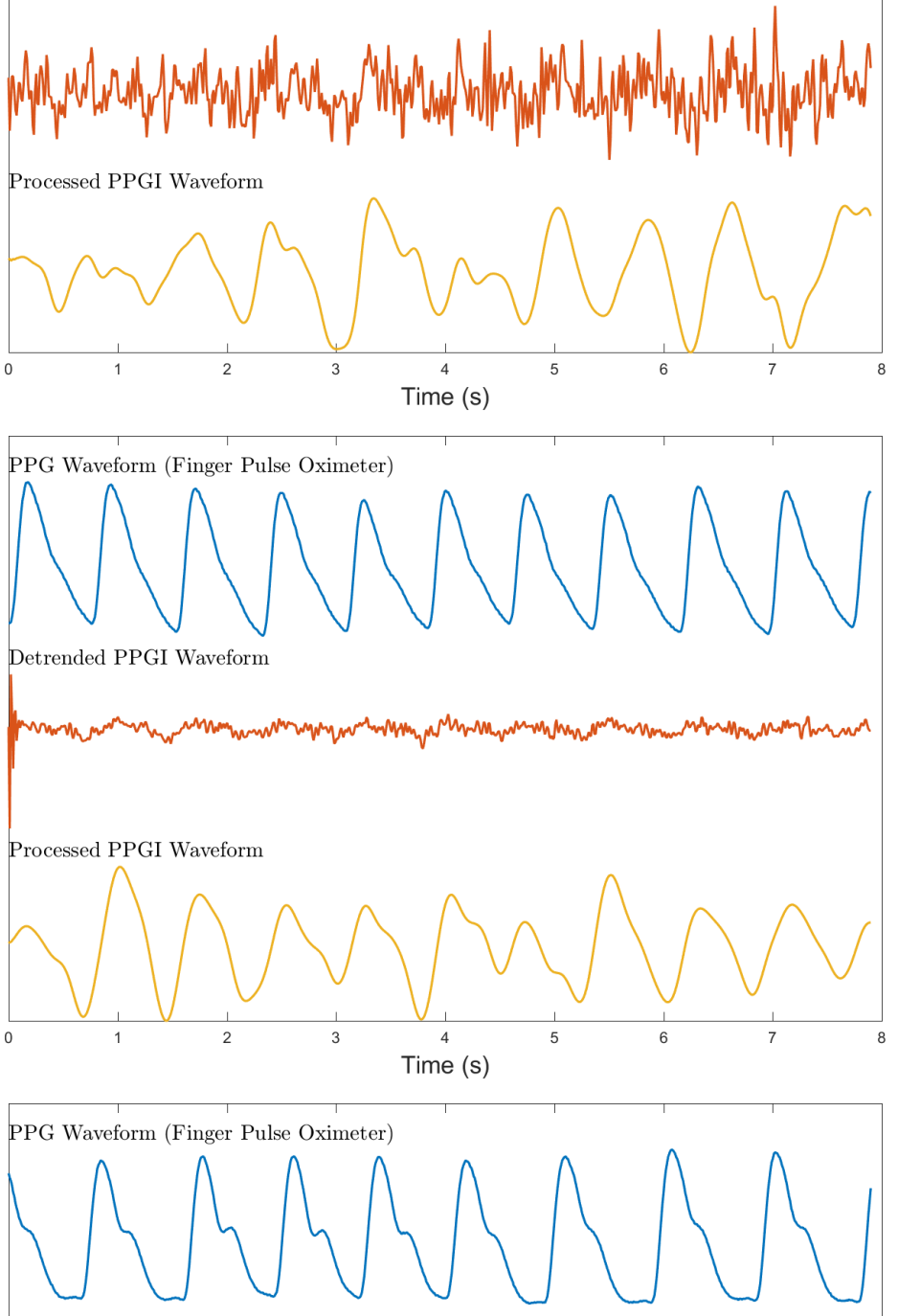

Detrended PPGI Waveform
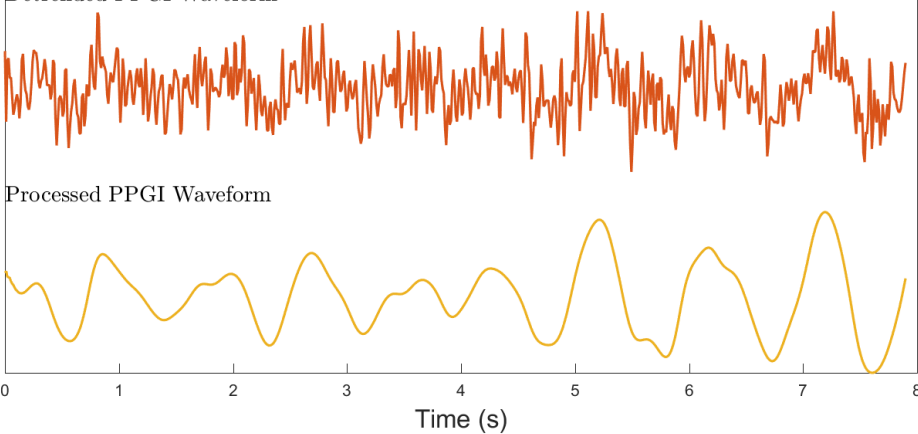

Fig. 3: Segments of a PPG waveform acquired from a finger pulse oximeter, the unprocessed PPGI waveform, and the PPGI waveform processed by our framework for six different participants.

the Natural Sciences and Engineering Research Council (NSERC) of Canada and the Ontario Ministry of Economic Development and Innovation.

\section{References}

[1] Murray, W., Foster, P. The Peripheral Pulse Wave: Information Overlooked. Journal of Clinical Monitoring (1996).

[2] Chwyl, B., Chung, A. G., Amelard, R., Deglint, J., Clausi, D. A., Wong, A. SAPPHIRE: Stochastically Acquired Photoplethysmogram for Heart Rate Inference in Realistic Environments. International Conference of Image Processing (2016).
[3] Tarvainen, M. P., Ranta-aho, P. O., Karjalainen, P. A. An Advanced Detrending Method with Application to HRV Analysis. IEEE Transactions on Biomedical Engineering (2002).

[4] Amelard, R., Hughson, L. R., Greaves, K. G., Pfisterer, K. J., Leung, J., Clausi, D. A., Wong, A. Non-Contact Hemodynamic Imaging Reveals the Jugular Venous Pulse Waveform. Preprint; arXiv:1604.05213v2 (2015).

[5] Wong, A., Wang., X. Y., A Bayesian Residual Transform for Signal Processing. IEEE Access (2015). 